\title{
Osteoporosis: Current review
}

\author{
HADI HEDAYATI, MD
}

JOHN J. ZUZGA, JR, DO

\begin{abstract}
The incidence of osteoporotic fracture, which commonly occurs in women at a site with large amounts of trabecular bone (spine, femur, or wrist), increases with age. Although the reduction of bone mass is an inevitable consequence of aging, appropriate prophylactic and therapeutic measures can significantly retard the development of osteoporosis, as well as decrease the cost, morbidity, and mortality that arises from complications of osteoporotic fractures. In this review, the pathophysiology, clinical presentation, diagnosis, prevention, and treatment of primary osteoporosis are discussed.
\end{abstract}

Osteoporosis, the most common nontraumatic disorder of the bones, is a major health problem to the elderly female population. As the amount of bone lessens with increasing age, the fracture rate increases. The disorder itself usually remains undetected until fracture occurs. However, there is effective prophylactic treatment for osteoporosis, and it is economically feasible to identify women at high risk so that early postmenopausal therapy may be instituted.

There is some confusion about the use of the terms osteopenia and osteoporosis. Osteopenia is used as a roentgenographic description of decreased bone density: It refers to an age-related physiologic loss of bone mass, which occurs both in cortical and trabecular bones and which predisposes the individual to fracture. In contrast, osteoporosis refers to the rapid or accelerated loss of bone mass prominent in the trabecular bones of postmenopausal women. Osteoporosis is associated with a high rate of vertebral crush fractures. In this discussion, we use the term osteoporosis to refer to both aging and postmenopausal bone loss, but a distinction between these two phenomena is made later in the paper.

\section{Bone cell physiology and metabolism}

The primary function of the skeleton is to provide a protective framework for other tissues and organs of the body. It also has an important role in calcium and phosphate homeostasis.

Understanding bone cell physiology and metabolism is essential in comprehending the development of osteoporosis. The cellular elements of the bone are osteoblasts, which produce bone matrix (osteoid), and osteoclasts, which are multinucleated cells responsible for bone resorption. Extracellular matrix of bone consists chiefly of collagen, type I, which binds to minerals, mainly calcium and phosphate. This leads to formation of hydroxyapatite crystals in bone.

Other elements, including magnesium, fluoride, and sodium, also are found in bone. These may play an important role in bone's structural and metabolic functions. Osteoblastic activity can be determined by measurement of serum alkaline phosphatase and proteins containing $\mu$-carboxyglutamic acid (Gla) or osteocalcin. Bone resorption or osteoclastic activity can be gauged by measuring hydroxyproline levels in the urine.

Cortical or compact bones comprise $80 \%$ of the total bones; the remaining $20 \%$ are spongy or trabecular bones.

Calcium is the major inorganic compound of bone matrix. One percent of the body's pool of calcium is exchangeable with extra cellular fluid. The serum calcium level is primarily under the influence of vitamin $\mathrm{D}$, parathyroid hormone $(\mathrm{PTH})$, calcitonin, and dietary intake.

Vitamin $\mathrm{D}_{3}$, or cholecalciferol, is synthsized mainly in the skin by nonenzymatic photolysis from 7-dehydrocholesterol and is carried to the liver by plasma transport protein. Exogenous sources of vitamin $\mathrm{D}_{3}$ are absorbed through the intestines, transported to the liver, and converted to 25-hydroxycholecalciferol $\left(25-\mathrm{OHD}_{3}\right.$ calcifediol $)$ by 25 hydroxylase enzyme(s). In the kidneys, $1 \tau$-hydrox- 
ylase enzyme converts $25-\mathrm{OHD}_{3}$ to active 1,25 dihydrocholecalciferol $\left(1,25(\mathrm{OH})_{2} \mathrm{D}_{3}\right.$, calcitriol. In addition to the kidneys, $1 \alpha$-hydroxylase is found in the placenta, bone, and, perhaps, sarcoid granuloma. PTH is required for this conversion.

The active form of vitamin $\mathrm{D}$ acts primarily on intestine to increase calcium absorption and also may act on bone to promote mineralization. At supraphysiologic levels, it also increases serum calcium by stimulating osteoclastic activity and inhibiting bone matrix synthesis by osteoblasts; this is particularly important when there is inadequate intake of calcium. The $25-\mathrm{OHD}_{3}$ level depends on the amount of $\mathrm{D}_{3}$ synthesis, or intake and reflect vitamin $\mathrm{D}$ stores in the body.

In normal elderly and postmenopausal osteoporotic patients, the concentration of serum 1,25 $(\mathrm{OH})_{2} \mathrm{D}_{3}$ falls, which results in a significant decrease in intestinal calcium absorption. Gallagher and associates ${ }^{1}$ found that administration of 1,25 $(\mathrm{OH})_{2}$ vitamin $\mathrm{D}$ in osteoporotic patients enhanced absorption of calcium from intestine.

In response to hypocalcemia, PTH is released to maintain the level of calcium in extracellular fluid by three mechanisms: (1) Increased absorption of calcium from the intestines by increased synthesis of $1,25(\mathrm{OH})_{2} \mathrm{D}_{3}$ in the kidney; (2) stimulation of osteoclasts and increased bone resorption; and (3) increased reabsorption of calcium in kidney tubules.

Chronic administration of glucocorticoids leads to a decrease in bone mass and retards skeletal growth. Glucocorticoids diminish absorption of calcium and phosphorous from the intestine and increase urinary excretion of calcium, which may result in stimulation of PTH secretion. Glucocorticoid excess decrease bone formation by inhibiting replication and differentiation of osteoblastic precursors in process of remodeling.

The hormone calcitonin, which is secreted from the C-cells of the thyroid gland, plays an important role in balancing the calcium level. Secretion is controlled by serum calcium, and the function of this hormone is opposite to that of PTH. Calcitonin inhibits bone resorption by decreasing activity of osteoclasts.

Several other hormones act on bone. Normal bone growth and remodeling require thyroid hormones. However, excess thyroid hormones can increase bone resorption and mild hypercalcemia can occur. The sex hormones estrogen, androgen, and progesterone also affect bone remodeling.

The prostaglandin E (PGE) series, when highly concentrated in the organ culture, inhibits bone collagen synthesis. When at a physiologic level, PGE seems to stimulate such synthesis. PTH stimulates the production of prostaglandin in bone, but vitamin $\mathrm{D}$ either has no effect or inhibits local PGE production. An example that excess prostaglandin acts locally as a pathologic mediator in development of periarticular osteoporosis in rheumatoid arthritis.

\section{Bone formation, resorption, and remodeling}

Bone is in a constant remodeling state during the entire life span. The net amount of bone present at any given time represents the balance between bone formation and resorption. Bone resorption is a function of osteoclasts and is regulated by PTH and $1,25(\mathrm{OH})_{2} \mathrm{D}_{3}$. These two hormones have receptors on osteoblasts, but not on osteoclasts. It appears that signals from osteoblasts are sent to osteoclasts to stimulate bone resorption. Osteoclasts have receptors for calcitonin, which inhibits bone resorption.

In normal bone, the rate of formation is equal to the rate of resorption. In osteoporosis, there is acceleration of bone resorption, decreased bone formation, or both. The balance between formation and resorption is disturbed with age. Generally, bone mass remains relatively unchanged until age 40 , when it begins to decline.

Bone mass is greater in men than in women at any given age. After age 40 , the rate of cortical bone loss in men is approximately .5\% to $.75 \%$ per year; in women, the rate is approximately $1.5 \%$ to $2 \%$ per year. After cessation of menstruation (menopause), there is an accelerated phase of bone loss. This phase lasts about 10 years and some women could lose a significant percentage of trabecular bone per year.

\section{Factors responsible for bone loss and osteoporosis}

Age and sex

Age-related bone loss is a multifactorial phenomenon. Elderly persons not only often consume less calcium than required, but intestinal absorption of calcium also is diminished with aging.

The active form of vitamin D is decreased with age; in osteoporotic individuals it is around the lower limits of normal. ${ }^{1,2}$

Initial studies ${ }^{3,4}$ suggest that concentration of calcitonin and its secretion is lower in women and declines with age. Since calcitonin inhibits bone resorption, which is the main skeletal defect in osteoporosis, deficiency of this hormone may contribute to pathogenesis of osteoporosis in elderly women. However, long-term calcitonin excess in patients with medullary carcinomia of thyroid or deficiency of calcitonin in patients with thyroidectomy did not alter bone mass. ${ }^{5}$

The decline in estrogen during menopause greatly increases the rate of bone loss, which leads 
to a higher incidence of osteoporosis and hip fractures in women as compared to men.

\section{Genetics}

Body size and the relative massiveness of bony elements are determined by hereditary factors.

A person with a smaller skeleton has a higher fracture risk than the same-aged individual with a large skeleton.

Those with fair skin have a higher risk of osteoporosis than darker-skinned persons. For example, Northern Europeans and Orientals are more at risk than others. Also, blacks have greater bone mass than whites.

\section{Physical activity}

Mechanical loading (weight bearing) is an important factor in maintaining bone mass, and its strength. Physical activity decreases with age, which leads to less bone mass. Obviously, a sedentary person is more likely to become osteoporotic than an active person who performs weight-bearing exercise.

\section{Nutrition}

During recent years, the importance of balanced nutrition with adequate calcium intake in maintaining bone mass has been stressed.

Calcium. Heaney and coworkers ${ }^{6}$ showed that calcium balance is linearly related to dietary calcium intake in premenopausal women using self-selected diets. Although premenopausal women require 1.0 to $1.2 \mathrm{~g}$ of calcium daily and postmenopausal women require $1.5 \mathrm{~g}$ a day, ${ }^{6,7}$ most women ingest only about $700 \mathrm{mg}$ of calcium per day. In premenopausal women this results in a negative calcium balance, which, because of the decrease in calcium absorption and the increase in urinary calcium excretion, is even more severe in postmenopausal women.

Protein. Excess protein intake results in a negative calcium balance. Sulfate, which is a byproduct of the metabolism of sulfur-containing amino acids, is excreted in urine. Either sulfate or its associated acid load to the kidneys will result in an increased glomerular filtration rate and decrease in tubular reabsorption of calcium. ${ }^{8}$

A high protein intake is associated with an excess phosphorous level. Phosphorous decreases urinary excretion of calcium, which compensates for the protein-induced calcium loss. However, because phosphorus increases fecal calcium loss, the net result is a negative calcium balance.

Caffeine. Caffeine increases both urinary and fecal loss of calcium.

\section{Alcholism}

In alcoholics, an inadequate intake of calcium resulting from poor nutrition, calcium malabsorption, and increased urinary calcium excretion, all contribute to bone loss.

\section{Smoking}

Women who smoke become menopausal earlier than nonsmokers, presumably because of the effect of smoking on circulating estrogen. In a study of postmenopausal women treated with exogenous estrogen, Jensen and coresearchers ${ }^{9}$ reported lower estrogenic levels in smokers than in nonsmokers.

Enhanced drug metabolism in smokers also has been reported. ${ }^{10}$ This extra degradation of estrogen and increased metabolic clearance rate are suggested as the causes of low estrogen levels in postmenopausal smokers who are being treated with exogenous hormones.

\section{Hormonal factors}

Estrogen may counteract the effects of PTH on bone resorption. When the estrogen level is deficient, the bone becomes more responsive to the effects of PTH.

Hyperthyroidism (endogenous or exogenous) has been associated with bone loss. Recently, Coindre and associates ${ }^{11}$ reported that bone mass is increased in the hypothyroid state, while replacement therapy to the euthyroid state results in cortical and trabecular bone loss.

\section{Methods of measuring bone mass}

Measurement of the rate of loss of bone mass in early postmenopausal periods, before osteoporosis is established in females at high risk, is essential. Several techniques have been used to evaluate and measure the bone mass and the rate of bone loss.

In clinical practice, osteopenia is a roentgenographic diagnosis defined as thinning of cortex and loss of trabeculae. However, by the time radiographic evidence of osteopenia is present, at least $30 \%$ to $50 \%$ of the bone mass is lost. Trabecular bone loss is more significant clinically because the risks of fracture of the spine, femoral neck, and wrist are increased greatly. X-ray of dorsal spine, lateral lumbar, and anteroposterior pelvic views will give the best diagnostic yield. The pelvic view should include the proximal part of the femur.

The following features should be looked for on the spinal film: (1) accentuation of vertical trabeculae, because the horizontal trabeculae are lost before the vertical trabeculae; (2) vertebral biconcavity, which usually is found in young persons with osteoporosis secondary to intervertebral disk expansion into the body of the vertebrae and which infrequently is seen in elderly persons whose disks 


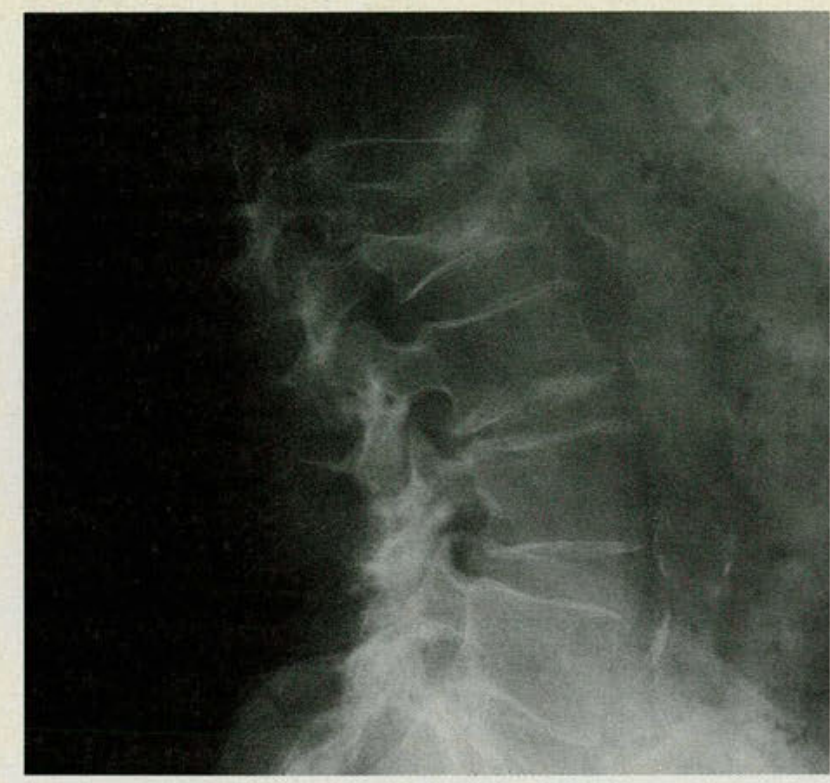

Fig 1. X-ray showing severe compression fracture of upper lumbar in osteoporosis.

have become degenerated and; (3) wedging (anterior height of vertebrae is less than the posterior height), which occurs predominantly in the dorsal spine (Fig 1).

On pelvic film, the calcar femorale should be evaluated. The thickness of cortical bones above the lesser trochanter is related to the risk of femoral neck fracture. The thickness normally is greater than $5 \mathrm{~mm}$. The trabecular structure of the hip appears as a weblike configuration on $\mathrm{x}$-rays of the hips that tend to decrease with osteoporosis. The Singh Index is an approximation of the trabecular bone at the proximal femur. It is graded on a six point scale; six (normal) to one (severe osteoporosis). Values of three or less greatly increase the risk of femoral neck fractures (Fig. 2).

\section{Cortical bone mass measurement}

Radiogrammetry. Cortical thickness is measured at the midpoint of the metacarpal bones. The difference between the total width and medullary width represents the cortical thickness.

Radiogrammetry is valuable for monitoring the rate of bone loss by a series of $x$-ray views of the second, third, and fourth metacarpal bones of the hand and measuring the cortical area at a fixed distance. The advantages of this technique are simplicity, availabilty of data, and reasonable cost. Disadvantages are limited accuracy and poor correlation with other cortical sites, trabecular bone mass, and total body calcium. Therefore, clinical application of this method is not recommended in face of availability of better techniques to measure the bone mass.

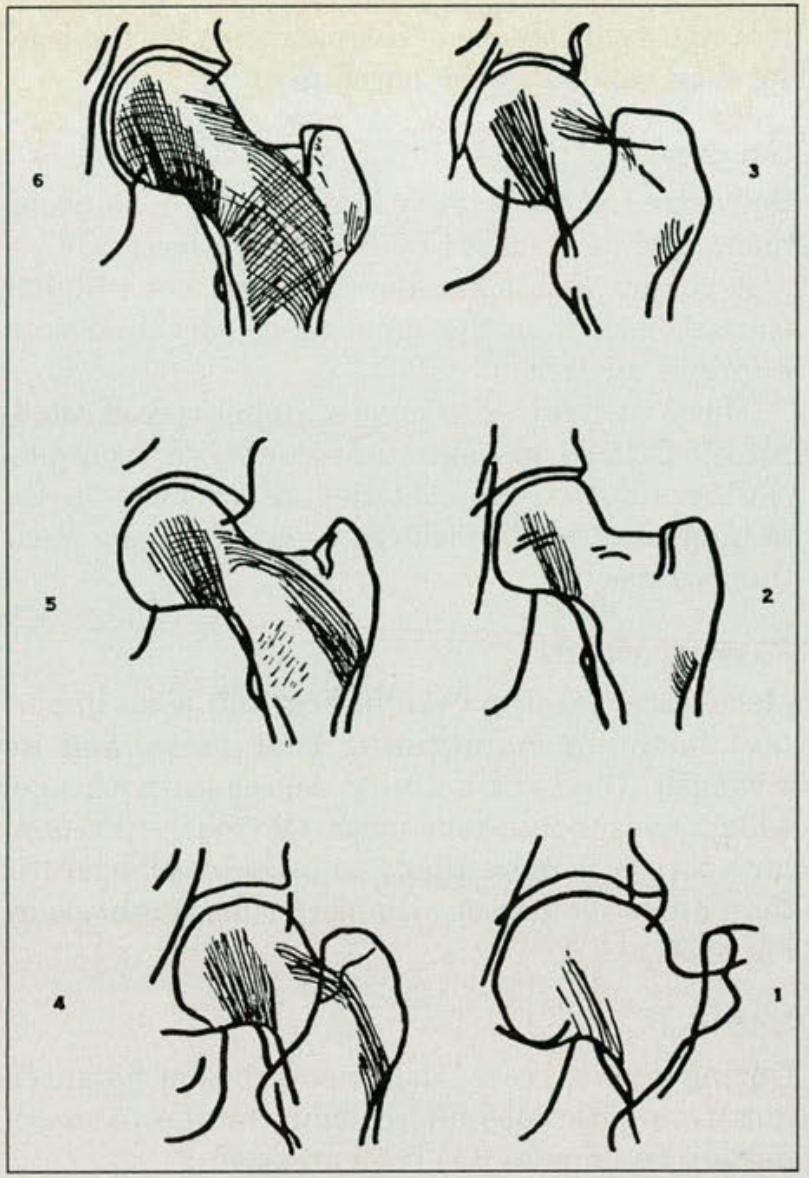

Fig. 2. Singh index analysis. Note the trabecular bone loss in lower index values. Three or less indicates presence of osteoporosis.

Single photon absorptiometry. The technique of photon absorptiometry uses a monoenergetic radioactive isotope, usually $\mathrm{I}^{125}$, to scan peripheral bones. The amount of skeletal minerals present is proportional to the change in beam intensity over the bone. The difference between photon absorption in bone and soft tissue allows measurement of the mineral content of bone. Most studies are of the radius and ulna. The test is accurate and can detect changes up to $1 \%$ to $2 \%$ per year loss in postmenopausal women.

This technique offers the advantages of good accuracy, precision, availability, modest cost, and little radiation exposure. There is good correlation with bone mass measured in the radius and other cortical sites, as well as with total body calcium. The changes found by this technique, however, are not a good index of changes in the trabecular bone of the spine and proximal femur.

\section{Trabecular bone mass measurement}

Aging decreases both compact bone and trabecular bone (total body bone mass). This phenomenon starts before middle age and is equal in men and 


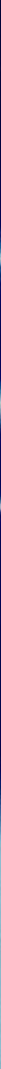

\section{(ciprofloxacin HCl/Miles)}

A REVOLUTIONARY

ORAL ANTIMICROBIAL 


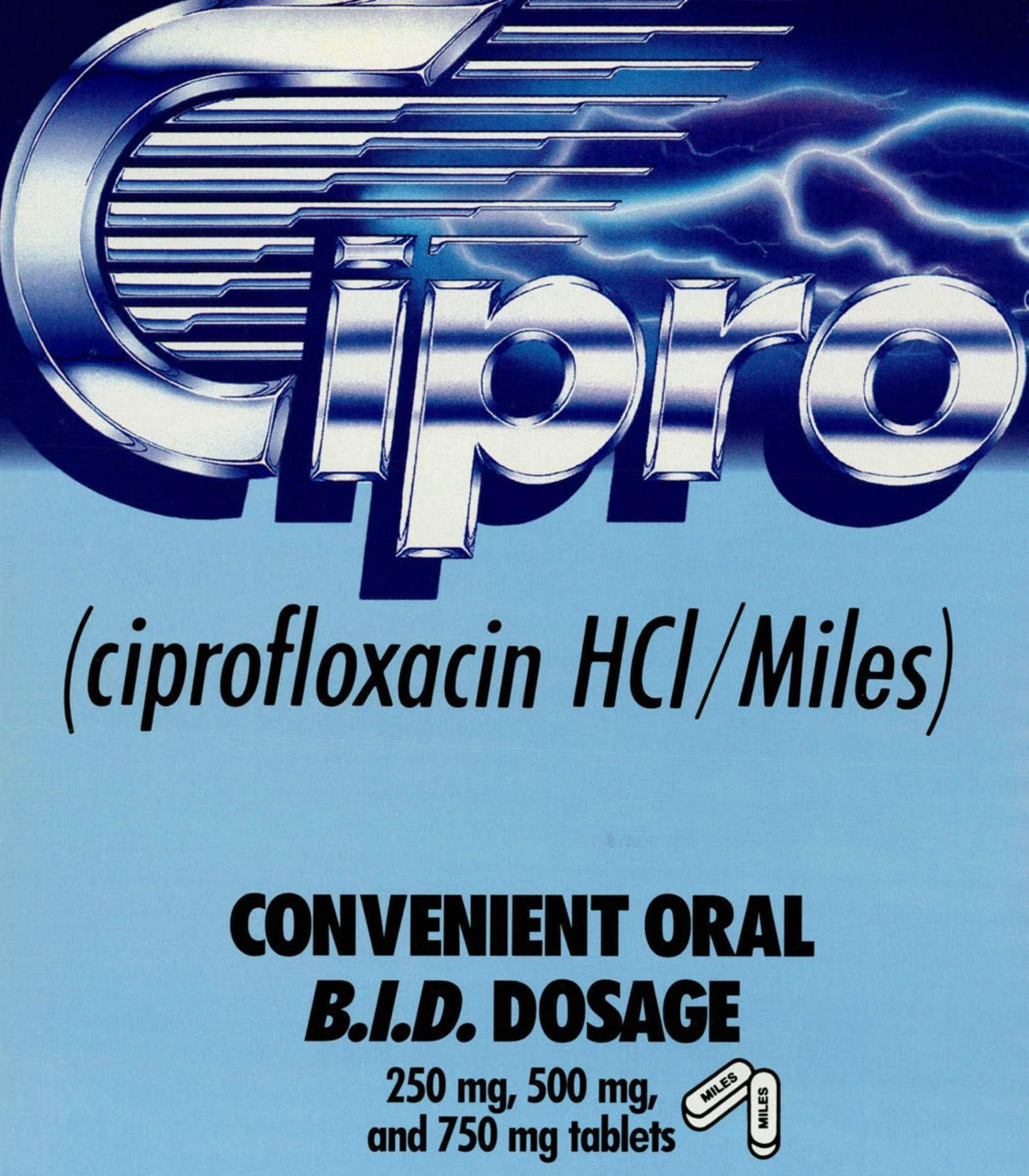




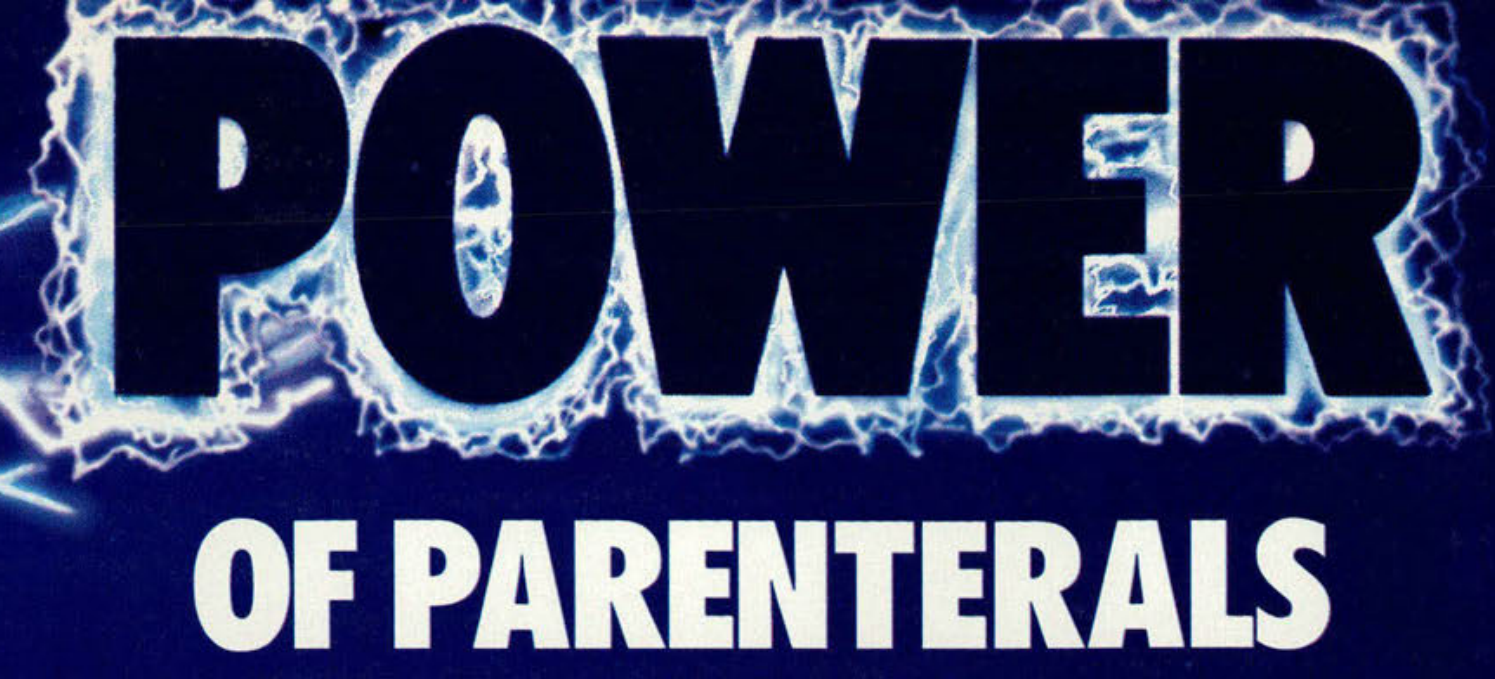

- Highly active in vitro against a broad range of gram-positive and gram-negative pathogens, including Pseudomonas aeruginosa and methicillin-resistant Staphylococcus aureus"

For treatment of infections in the: - lower respiratory tract - urinary tract
- skin/skin structure - bones and joints

\section{Well tolerated-low incidence of side effects}

For further information, contact the Miles Information Service:

1-800-642-4776. (In VA, call collect: 703-391-7888.)

* In vitro activity does not necessarily imply a correlation with in vivo results.

Due to susceptible strains of indicated pathogens. See indicated organisms in Prescribing Information.

CIPRO ${ }^{*}$ SHOULD NOT BE USED IN CHILDREN, ADOLESCENTS, OR PREGNANT WOMEN

A history of hypersensitivity to ciprofloxacin is a contraindication to its use. A history of hypersensitivity to other quinolones may also contraindicate the use of ciprofloxacin.

Before prescribing Cipro ${ }^{\circledR}$, please see Brief Summary of Prescribing Information.

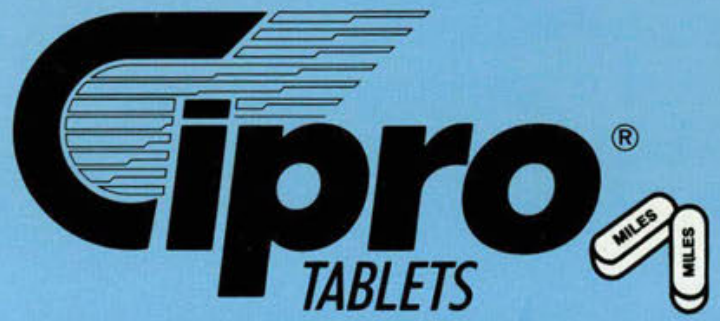

THE POWER OF PARENTERALS THE FREEDOM OF ORAL DOSAGE 


\section{Gipro: (ciprofloxacin $\mathrm{HCl} /$ Miles) \\ CONVENIENT B.I.D. DOSAGE Dosage guidelines Mild/Moderate Infections*: $500 \mathrm{mg}$ q12h Severe/Complicated Infections*: $750 \mathrm{mg}$ q12h}

\author{
CIPRO ${ }^{\circ}$ \\ (ciprofloxacin hydrochloride/Miles) \\ TABLETS \\ BRIEF SUMMARY \\ CONSULT PACKAGE INSERT FOR FULL PRESCRIBING INFORMATION \\ INDICATIONS AND USAGE \\ $\mathrm{Cipro}^{*}$ is indicated for the treatment of infections caused by susceptible strains of the designated micro
} organisms in the conditions listed below.

Lower Respiratory Infections caused by Escherichia coli, Klebsiella pneumaniae, Enterobacter cloacae Proteus mirabilis, Pseudomonas aeruginosa, Haemophilus influenzae, Haemophilus parainfluenzae, and Strep lococcus pneumoniae.

Skin and Skin Structure Infections caused by Escherichia coli, Klebsiella pneumoniae, Enterobacter cloacae Proteus mirabilis, Proteus vulgaris, Providencia stuartii, Morganella morganii, Citrobacter freundi. Pseudomonas aeruginosa, Staphylococcus aureus ipenicillinase and nonpenicillinase-producing strains Staphylacoccus epidermidis, and Streptococcus pyogenes

Bone and Joint Infections caused by Enterobacter cloacae, Serratia marcescens, and Pseudomonas aeruginosa.

Urinary Tract Infections caused by Escherichia coli, Klebsiella pneumoniae, Enterobacter cloacae, Serratia marcescens, Proteus mirabilis, Providencia rettgeri, Morganella morganii, Citrobacter diversus, Citrobacter freundii. Pseudomonas aeruginosa. Staphylococcus epidermidis, and Streptococcus faecalis.

Infectious Diarrhea caused by Escherichia coli lenterotoxigenic strains! Campylobacter jejuni, Shigella flexneri* and Shigella sonnei* when antibacterial therapy is indicated

Efficacy for this organism in this organ system was studied in fewer than 10 infections

Appropriate culture and susceptibility tests should be performed before treatment in order to isolate and identify organisms causing infection and to determine their susceptibility to ciprofloxacin. Therapy with Cipro" may be initiated before results of these tests are known; once results become available appropriate therap should be continued. As with other drugs, some strains of Pseudomonas aeruginosa may develop resistance fairly rapidly during treatment with ciprofloxacin. Culture and susceptibility testing performed periodically during therapy will provide information not only on the therapeutic effect of the antimicrobial agent but also on the possible emergence of bacterial resistance. CONTRAINDICATIONS

A history of hypersensitivity to ciprofloxacin is a contraindication to its use. A history of hypersensitivity to othe quinolones may also contraindicate the use of ciprofloxacin.
WARNINGS

CIPROFLOXACIN SHOULD NOT BE USED IN CHILDREN. ADOLESCENTS, OR PREGNANT WOMEN. The ora administration of ciprollaxacin caused lameness in immature dogs. Histopathological examination of the weight bearing joints of these dogs revealed permanent lesions of the cartiage. Related drugs such as nalidixic acid cinoxacin, and nortloxacin also produced erosions of cartilage of weight-bearing joints and other signs arthropathy in immature animals of various species ISEE ANIMAL PHARMACOLOGY SECTION IN FULL PRESCRIBING INFORMATION

\section{PRECAUTIONS}

General: As with other quinolones, ciprofloxacin may cause central nervous system (CNS) stimulation, which may lead to tremoc, restlessness, ightheadedness, confusion, and very rarely to hallucinations or convulsi seizures. Therefore, ciprofloxacin should be used with caution in patients with known or suspected CNS disorders, such as severe cerebral arteriosclerosis or epilepsy. or other factors which predispose to seizures (SEE ADVERSE REACTIONS

Quinolones may also cause anaphylactic reactions and cardiovascular collapse. Anaphylactic reactions may require epinephrine and other emergency measures.

Crystals of ciprofloxacin have been observed rarely in the urine of human subjects but more frequently in the urine of laboratory animals. Crystalluria related to ciprofloxacin has been reported only rarely in man because human urine is usually acidic. Patients receiving ciprofloxacin should be well hydrated, and alkalinity of the urine necessary for patients with impairment of renal function (SEE DOSAGE AND ADMINISTRATION SECTION IN FULL PRESCRIBING INFORMATION

Drug Interactions: Concurrent administration of ciprofloxacin with theophylline may lead to elevated plasm concentrations of theophyline and prolongation of its elimination half-life. This may result in increased risk of theophylline-related adverse reactions. If concomitant use cannot be avoided, plasma levels of theophylline should be monitored and dosage adjustments made as appropriate.

Antacids containing magnesium hydroxide or aluminum hydroxide may interfere with the absorption of ciprofloxacin, resulting in serum and urine levels lower than desired: concurrent administration of these agents with ciprofloxacin should be avoided.

Probenecid interferes with the renal tubular secretion of ciprofloxacin and produces an increase in the level of ciprofloxacin in the serum. This should be considered if patients are receiving both drugs concomitantly

As with other broad-spectrum antibiotics, prolonged use of ciprofloxacin may result in overgrowth of nonsusceptible organisms. Repeated evaluation of the patient's condition and microbial susceptibility testing is essential. If superinfection occurs during therapy appropriate measures should be taken.

Information for Patients: Patients should be advised that ciprofloxacin may be taken with or without meals The preferred time of dosing is two hours after a meal. Patients should also be advised to drink fluids liberally and not take antacids containing magnesium or aluminum concomitantly of within two hours after dosing. Ciprofloxacin may cause dizziness or lightheadedness: therefore patients should know how they react to this drug before they operate an automobile or machinery or engage in activities requiring mental alertness of coordination.

Carcinogenesis, Mutagenesis, Impairment of Fertility: Eight in vitro mutagenicity tests have been conducted with ciprofloxacin and the test results are listed below:

Salmone

E. coli DNA Repair Assay (Negative)

Mouse Lymphoma Cell forward Mutation Assay (Positive)

Chinese Hamster $\mathrm{V}_{79}$ Cell HGPRT Test (Negative)

Syrian Hamster Embryo Cell Transformation Assay (Negative)

Saccharomyces cerevisiae Point Mutation Assay (Negative)

Saccharomyces cerevisiae Mitotic Crossover and Gene Conversion Assay (Negative)

Rat Hepatocyte DNA Repair Assay (Positive)

Thus, two of the eight tests were positive, but the following three in vivo test systems gave negative results: Rat Hepatocyte DNA Repair Assay

Micronucleus Test (Mice)

g-term carcinogenicity studies in animals have not yet been completed

Pregnancy-Pregnancy Category C: Reproduction studies have been performed in rats and mice at doses up to six times the usual daily human dose and have revealed no evidence of impaired fertility or harm to the fetus due to ciprofloxacin. In rabbits, as with most antimicrobial agents, ciprofloxacin 130 and $100 \mathrm{mg} / \mathrm{kg}$ orally) produced gastrointestinal disturbances resulting in maternal weight loss and an increased incidence of abortion.
No teratogenicity was observed at either dose. After intravenous administration, at doses up to $20 \mathrm{mg} / \mathrm{kg}$ maternal toxicity was produced, and no embryotoxicity or teratogenicity was observed. There are, howeve
adequate and well-controlled studies in pregnant women. SINCE CIPROFLOXACIN. UKE OTHER DRUGS IN
CLASS. CAUSES ARTHROPATHY IN IMMATURE ANIMALS. IT SHOULD NOT BE USED IN PREGNANT WO CLASS, CAUSES ARTHROPATHY IN IMMATURE ANIMALS, IT SHOULD NOT BE USED IN PREGNANT WO (SEE WARNINGS)

Nursing Mothers: It is not known whether ciprofloxacin is excreted in human milk; however, it is known ciprofloxacin is excreted in the milk of lactating rats and that other drugs of this class are excreted in human Because of this, and because of the potential for serious adverse reactions from ciprofloxacin in nursing infar decision should be made to discontinue nursing or to discontinue the drug. taking into account the importan the drug to the mother

Pediatric Use: Ciprofloxacin should not be used in children because it causes arthropathy in immature an [SEE WARNINGS]

\section{ADVERSE REACTIONS}

forloxacin is generally well tolerated During chinicat investigation, 2799 patients teceived 2868 cours the drug. Adverse events that were considered likely to be drug related occurred in $7.3 \%$ of courses, pos related in $9.2 \%$, and remotely related in $3.0 \%$. Ciprofloxacin was discontinued because of an adverse eve $3.5 \%$ of courses, primarily involving the gastrointestinal system $1.5 \%$. skin $10.6 \%$. and central nervous sy $10.4 \%$

The most frequently reported events, drug related or not, were nausea $(5.2 \%)$ diarrhea $(2.3 \%)$ vomiting 12 adominal pain/discomfort $(1.7 \%$, headache $(1.2 \%)$ restlessness $(1.1 \%)$ and rash $11.1 \%$

Additional events that occurred in less than $1 \%$ of ciprofloxacin courses are listed below. Those typic

GASTROINTESTINAL: (See above), painful oral mucosa, oral candidiasis, dysphagia, intestinal perfora

gastrointestinal bleeding. tions, manic reaction irritability, tremor, ataxia, convulsive seizures, lethargy, drowsiness, weak

malaise, anorexia, phobia, depersonalization, depression, paresthesia.
SKIN/HYPERSENSITIVITY: (See above), pruritus, urticaria, photosensitivity, flushing, fever, ct angioedema, edema of the face, neck, lips, conjunctivae or hands, cutaneous candidiasis, hyperpigm tion, erythema nodosum.

Allergic reactions ranging from urticaria to anaphylactic reactions have been reported.

SPECIAL SENSES: blurred vision, disturbed vision, (change in color perception, overbrightness of lig decreased visual acuity, diplopia, eve pain, tinnitus, bad taste.

MUSCU U RENAL/UROGENITAL

myocardial infarction, cardiopum monary arrest, cerebral thior

pulmonary embolism.

Most of these events were described as Intinued, and required no treatment.

In several instances, nausea, vomiting, tremor, restlessness, agitation, or palpitations were judge investigators to

Adverse Laboratory Changes: Changes in laboratory parameters listed as adverse events without rega

drug relationship.

Hepatic - Elevations of: AIT (SGPT) (1.9\%) AST ISGOT) (1.7\%), alkaline phosphatase $(0.8 \%)$ LDH 10 .

serum bilirubin $10.3 \%$

Hematologic - eosinophilia $(0.6 \%)$ leukopenia $(0.4 \%)$ decreased blood platelets $(0.1 \%)$ elevated b platelets $10.1 \%)$ pancytopenia $(0.1 \%)$

CRYSTALLUURIA, CYIINDRURIA. AND HEMATURIA HAVE BEEN REPORTED

Other changes occurring in less than $0.1 \%$ of courses were: Elevation of serum gammaglutamyl transfer elevation of serum amylase, reduction in blood glucose, elevated uric acid, decrease in hemoglobin, ane bleeding diathesis, increase in blood monocytes, and leukocytosis.

Information on overdosage in humans is not available. In the event of acute overdosage, the stomach shoul emptied by inducing vomiting or by gastric lavage. The patient should be carefully observed and given suppo treatment Adequate hydrations beric reactions from overdos function is compromised.

\section{DOSAGE AND ADMINISTRATION}

The usual adult dosage for patients with urinary tract infections is $250 \mathrm{mg}$ every 12 hours. For patients complicated infections caused by organisms not highly susceptible, $500 \mathrm{mg}$ may be administered every $12 \mathrm{~h}$ Respiratory tract infections, skin and skin structure infections, and bone and joint infections may be tre With $500 \mathrm{mg}$ every 12 hours for more severe or complicated infections, a dosage of $750 \mathrm{mg}$ may be given eve hours.

The recommended dosage for infectious diarrhea is $500 \mathrm{mg}$ every 12 hours.

In patients with renal impairment, some modification of dosage is recommended ISEE DOSAGE ADMINISTRATION SECTION IN FULL PRESCRIBING INFORMATION HOW SUPPLED

Cipro (ciprofloxacin HCI/Miles) is available as tablets of $250 \mathrm{mg}$. $500 \mathrm{mg}$. and $750 \mathrm{mg}$ in bottles of 50 , a Unit-Dose packages of 100 (SEE FULL PRESCRIBING INFORMATION FOR COMPLETE INFORMATION)

*Due to susceptible strains of indicated pathogens. See indicated organisms in Prescribing Information.

For further information, contact the Miles Information Service: 1-800-642-4776. In VA. call collect: 703-391-7888.

COMMITTED TO THERAPEUTIC EFFICIENC

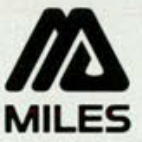

c) October 1988 , Miles Inc.

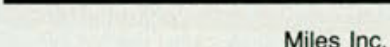

Pharmaceutical Divisi 400 Morgan Lane

West Haven, CT 0651 
women (approximately $1 \%$ annually) except postmenopause, when bone loss is accelerated.

Because of higher fracture risk, measurement of trabecular bone mass in the spine and femoral neck is more important clinically than radius and metacarpal measurement. There are two techniques for trabecular measurement:

Dual photon absorptiometry. This technique measures bone mass within the femur and lumbar spine, including body and transverse process. The longitudinal measurement of bone density is valuable in the early postmenopausal phase for prophylactic treatment of patients with accelerated bone loss. The cost of this method is reasonable, and the radiation dose is low.

Computed tomography(CT). All available CT machines use the $\mathrm{x}$-ray as the source of radiation. This technique is suitable for the measurement of thicker body areas, including the spine, femur, and humerus. CT also is accurate for large, compact bones, such as the radius. This technique costs more and the radiation dose is higher than dual photon absorptiometry.

Currently, the most cost-effective technique for measuring bone mass and response to therapy is the dual photon beam. It should be pointed out, however, that this technique does not differentiate bone loss from osteomalacia, hyperthyroidism, hyperparathyroidism, malignancy, or hypercortisonism. It only identifies the presence of decreased mass.

\section{Clinical features, diagnosis, and differential diagnosis}

There are several symptoms that should alert the clinician to osteoporosis. There are three prinici-

\begin{tabular}{|l|}
\hline TABLE 1. DIFFERENTIAL DIAGNOSIS OF OSTEOPENIA IN ADULTS. \\
\hline Primary osteopenia/osteoporosis \\
Postmenopausal osteoporosis (type I) \\
Senile osteoporosis (type II) \\
Idiopathic osteoporosis \\
Immobilization \\
Secondary osteopenia \\
Endocrine disease \\
Hyperparathyroidism \\
Hyperthyroidism \\
Glucocorticoid overproduction \\
Drug-induced disease \\
Glucocorticoid therapy \\
Heparin therapy \\
Anticonvulsant therapy \\
Osteomalacia \\
Miscellaneous diseases \\
Multiple myeloma or other malignancy \\
Renal failure \\
Malabsorption \\
Rheumatoid arthritis \\
Alcoholism
\end{tabular}

ple sites for osteoporotic fractures--the vertebrae, the femur, and the distal radius. These tend to occur with minor trauma or in the absence of trauma.

Dorsal kyphosis, pain, and loss of height often are associated with spinal osteoporosis. The 12 thoracic and first lumbar verbrae commonly are fractured. The pain of compression fracture usually is of sudden onset; it is sharp, and it becomes worse with movement and weight-bearing. The laboratory data (including serum calcium phosphorous and alkaline phosphatase values), are normal, except when fracture is present, in which case the alkaline phosphatase level may be elevated.

The differential diagnosis of osteopenia is illustrated in Table 1.

The clinical diagnosis of senile, postmenopausal, or idiopathic osteoporosis is essentially one of exclusion. It is important to perform certain tests to exclude diseases other than primary osteoporosis (Table 2).

It is valuable to characterize primary osteoporosis as postmenopausal (type I) or senile (type II). The differences between these two entities is outlined in Table 3.

Osteoporosis and osteoarthritis are common disorders that increase in frequency with age. Coexistence of these two entities is not common. Degenerative disk disease, however, may be seen in either disease. In osteoporosis, the rate of bone loss is greater than normal. In osteoarthritis, there is slower or no bone loss with age.

Estrogen has no effect on the prevention of osteoarthritis, while its use in early stages of postmenopause prevents osteoporosis. Growth hormones may be involved in the pathogenesis of osteoarthritis, and higher than normal levels are found in that disease, but not in osteoporosis.

In addition to genetic and hormonal factors, the observation of qualitative and quantitative differences in the protein matrix of bone in osteoarthritis and osteoporosis indicates that these two entities are not simply age-related phenomena. ${ }^{12}$

\section{Prevention and treatment of postmenopausal osteoporosis}

The goals of therapy are to maintain normal or near-normal bone mass, to prevent further bone

TABLE 2. LABORATORY PROCEDURES IN EVALUATION OF

OSTEOPOROSIS.

Determination of serum calcium, phosphorus, and alkaline phosphatase values

Protein electrophoresis

Measurement of erthrocyte sedimentation rate

Thyroid function test

Measurement of parathyroid hormone level 
loss in patients who already are osteoporotic, and to diminish the pain and disability.

As far as prevention for early postmenopausal women is concerned, the clinician should evaluate the risk factors by the history (Table 4), and, if the patient is categorized as being in a high-risk group, vertebral bone densiometry is indicated. Prophylactic therapy should be given to patients with low densiometric values.

Once the diagnosis of primary osteoporosis has been established, the next steps are to determine the severity of the disease and to institute a treatment program. At the present time, all treatment modalities either prevent further bone loss, or, at best, cause a small positive bone balance. No current treatment program will reverse the loss that has occurred.

Therapy is based on exercise, calcium, and vitamin D supplementation, estrogen replacement, and, in exceptional cases, fluoride or calcitonin use. First, we will outline the strategy for prevention and treatment of osteoporosis (Tables 5 and 6). Later, the management of acute compression fracture will be discussed.

\section{Physical activity}

Adequate physical activity is needed to maintain bone mass and bone strength. One theory states that pulling and pushing of tendon muscles on bone increases the electrical current, which may help to maintain bone structure. The other theory suggests that increased activity may help to improve calcium absorption. Whichever is true, the important questions are how much and what kind of physical activity is necessary to maintain bone density.

Intense physical activity in young women who become amenorrheal results in a significant reduction of bone mass as compared to those who are eumenorrheic. ${ }^{13}$ The risk of trauma also increases with great physical activity.

Marked osteoporosis is seen in immobilized patients. When the patient becomes mobile and active, original bone density returns. ${ }^{14}$

Studies demonstrate significant bone loss in astronauts in extended space flight, even with aerobic exercises. This indicates that exercise should be performed against gravity and should be weightbearing.

\begin{tabular}{|c|c|c|}
\hline Parameter & Postmenopausal & Senile \\
\hline Age $(y r)$ & 55-75 & $70-85$ \\
\hline Female/male ratio & $6: 1$ & $2: 1$ \\
\hline Bone loss & Trabecular > cortical & Cortical $=$ trabecular \\
\hline Site of fractures & Vertabrae, wrist & Hip, vertebrae \\
\hline Main etiology & Lack of estrogen & Aging \\
\hline
\end{tabular}

Daily physical activity and exercise should be encouraged, particularly for postmenopausal women. For aging patients, the best activity is daily walking or swimming. Running should be avoided by elderly persons with osteoporosis. It may lead to compression fracture of vertebrae.

\section{Calcium supplementation}

Adequate calcium intake is crucial for attaining and maintaining optimum bone mass, which occurs at ages 25 to 35 . Individuals deficient in calcium have less bones mass than that expected for age and are at higher risk for developing osteoporosis.

Epidemiologic data ${ }^{15}$ indicate that calcium provides significant protection against age-related bone loss and hip fracture. It seems reasonable to educate adolescents about adequate calcium intake, physical activity, and avoidance of smoking and excess caffeine and alcohol consumption in order to optimize bone mass during bone growth.

Heaney and coauthors ${ }^{7}$ studied three groups of postmenopausal women and compared the effects of estrogen treatment, calcium supplementation, and no therapy. They found that both estrogen and calcium therapy decreased the rate of bone resorption (as measured by radio-labeled calcium kinetic studies). Riggs and coauthors ${ }^{16}$ recently reported the absence of significant correlation between dietary calcium intake and changes of bone mineral density in normal women, but the question of the value of high-dose calcium use for osteoporotic patients remains open.

However, the daily requirement of calcium is about 1.0 to $1.2 \mathrm{~g}$ for premenopausal women, and $1.5 \mathrm{~g}$ for postmenopausal women. ${ }^{6,7}$ With the usual dietary intake of calcium for most women totaling about $700 \mathrm{mg} /$ day, supplemental calcium obviously is needed to avoid negative calcium balance. Prescribing calcium requires that one know how much

TABLE 4. RISK FACTORS FOR OSTEOPOROSIS.

Aging

Menopause (surgical or natural)

Female sex, white race, fair skin

Small frame

Family history of osteoporosis

Lack of physical activity

Low calcium intake

Smoking

Excess consumption of caffeine, alcohol, protein

TABLE 5. STRATEGY FOR PREVENTION OF OSTEOPOROSIS IN POSTMENOPAUSAL WOMEN.

Exercise

Estrogen and progesterone therapy

Calcium supplementation

Elimination of risk factors if possible 
elemental calcium is present in each preparation. Calcium carbonate has $40 \%$ elemental calcium, calcium phosphate has $31 \%$, and calcium gluconate has only $9 \%$. Calcium is best absorbed if it is taken in 3 divided doses.

Determination of serum calcium and phosphorous values and a 24-hour urine analysis for calcium, phosphorous, and creatinine are recommended before therapy. During calcium supplementation, encourage fluid intake and monitor the patient's serum and urine calcium once or twice yearly.

\section{Vitamin D supplementation}

It is reported ${ }^{1,2}$ that the serum concentration of 1,25 $(\mathrm{OH})_{2} \mathrm{D}_{3}$ is lower in patients with osteoporosis than in age-matched individuals without bone disease. Other studies indicated that vitamin $\mathrm{D}$ alone has no significant effect on calcium balance and bone mass, but when it was used in combination with calcium and hormones, there was significant improvement in the parameters assessed.

There has been evidence that $1,25(\mathrm{OH})_{2} \mathrm{D}_{3}$ improves the intestinal absorption of calcium in postmenopausal women. It is evident that plasma level of $25-\mathrm{OHD}_{3}$ decreases with age by $50 \% \cdot{ }^{17}$ Plasma $1,25(\mathrm{OH})_{2} \mathrm{D}_{3}$ concentration is lower in elderly persons. This contributes to the inability of the aging kidney to synthesize $1,25(\mathrm{OH})_{2} \mathrm{D}_{3}$ caused by decrease in $1 \tau$-hydroxylase activity and is a factor for pathogenesis of senile osteoporosis. ${ }^{18}$ The current supplement recommendation for vitamin $\mathrm{D}$ is 400 to $800 \mathrm{IU}$ daily.

\section{Estrogen therapy}

Normal ovarian function is required to retain bone mass. The mechanism by which estrogen can inhibit bone resorption is not well understood. There has been no evidence of estrogen receptors in bone; therefore, it had been thought that the effects of estrogen on bone were mediated indirectly. Recently, however, Eriksen and colleagues ${ }^{19}$ demonstrated evidence of estrogen receptors in osteoblasts, which indicates that estrogen may act directly on bone.

It is believed that estrogen prevents postmenopausal osteoporosis by reducing bone resorption and that there is a secondary decrease in bone formation; thus, bone mass is kept constant. Lindsay and associates ${ }^{20}$ showed that progesterone therapy given alone prevents bone loss without decreasing bone resorption, which indicates that progesterone increases bone formation. Other possible mechanisms for estrogen reducing bone resorption are as follows: decreased bone sensitivity to PTH; calcitonin secretion stimulation; and increased absorption of calcium from the gastrointestinal system.
TABLE 6. TREATMENT STRATEGY FOR SYMPTOMATIC OSTEOPOROSIS IN POSTMENOPAUSAL WOMEN.

Basic treatment

Exercise

Estrogen and progesterone therapy

Calcium supplementation (1500 mg daily)

Vitamin D supplementation (400 to 800 IU daily)

(If new fracture occurs, add fluoride supplementation, 40 to $80 \mathrm{mg} /$ day.)

Under the following conditions; add Calcitonin (Calcimar), 100 units daily (plus vitamin D calcium):

Estrogen therapy is contraindicated Patient is more than 15 years postmenopausal Severe osteoporotic pain is present

Studies show that hormone therapy prevents progression of bone reduction after menopause, ${ }^{21}$ and rapid bone loss begins as soon as estrogen therapy is stopped. ${ }^{22}$ Women treated within six years of menopause with either daily estrogen or cyclic estrogen and progesterone were able to maintain bone mass, and their fracture rate was decreased. ${ }^{23,24}$

All women should be evaluated prior to hormone therapy with a complete history and physical examination, complete blood cell count, urinalysis, mammography, pelvic examination, and Papanicolaou smear.

Postmenopausal hormone therapy in a woman with an intact uterus appears to be safer if cyclic administration of estrogen combined with progesterone is used (estrogen 25 days, progesterone 10 days, no treatment 5 days). Estrogen therapy should be avoided if any of the conditions listed in Table 7 is present. The recommended dosage is $0.6 \mathrm{mg}$ of estrogen per day, but as low a dose as $0.3 \mathrm{mg}$ per day has been reported to give good results. ${ }^{25}$

There are several known or potential risks with conjugated estrogen therapy (Table 8 ). There is a statistically significant increase in the incidence of endometrial cancer in patients treated with conjugated estrogen only. ${ }^{26}$ For patients taking daily estrogen therapy, the risks range from two to eight times greater than that of age-matched women without hormone therapy; with cyclic estrogen/progesterone therapy, risks range from 1.5 to 2 times that of untreated women. ${ }^{27}$ There is evidence, however, that the use of oral contraceptives containing estrogen and progesterone decreases the risk of endometrial cancer. ${ }^{28}$ It is possible that the progesterone protects the endometrium from the proliferative effect of estrogen.

It has been believed that endometrial cancer associated with estrogen is localized, not aggressive, 
TABLE 7. CONTRAINDICATIONS TO ESTROGEN THERAPY.

History of endometrial or breast cancer

Benign breast disease

Unexplained vaginal bleeding

Acute liver disease or chronic abnormal liver function

Acute vascular thrombosis

Uncontrolled hypertension

TABLE 8. RISKS OF ESTROGEN THERAPY.

Endometrial neoplasm

Breast cancer (?)

Cardiovascular disease(?)

Cholelithiasis

Hypertension

Venous thromboembolism

and that the risks diminish when therapy is discontinued. ${ }^{29,30,31}$ Later, it was reported ${ }^{32}$ that longterm use of conjugated estrogen therapy increases the risk of both localized and widespread endometrial cancer, and that the risk remains high for many years after therapy has been discontinued.

Yearly follow-up with pelvic examination and Papanicolaou smear is recommended. Endometrial biopsy should be performed if unusual bleeding occurs during the course of therapy. Uterine bleeding occurs in $50 \%$ to $60 \%$ of postmenopausal patients with an intact uterus who are receiving cyclic estrogen/progesterone therapy, which may lead to discontinuation of the medication by the patient, particularly the elderly patient.

There is no conclusive evidence that estrogen therapy increases the risk of breast cancer or that it offers protection against breast cancer. The Boston Collaborative Drug Surveillance Program ${ }^{33}$ showed no significant relationship between postmenopausal estrogen therapy and breast cancer. Another report ${ }^{34}$ suggests that oral contraceptive therapy does not increase the risk of breast cancer in women.

Alkjaersig and associates ${ }^{35}$ reported an increased risk of venous thrombosis in association with oral contraceptive use. Other authors ${ }^{36}$ showed no increase in venous thromboembolism in postmenopausal women treated with a combination of estrogens and progesterone. We recommend that estrogen replacement be avoided in women with previous thrombotic episodes.

It is possible that estrogen may induce hypertension by retention of sodium and that it may increase plasma renin activity in some cases. The incidence of cholelithiasis increases by 2.5 -fold in postmenopausal women receiving estrogen therapy. ${ }^{33}$

The effect of estrogen on the cardiovascular system is controversial. Estrogen influences the se- rum lipid level. It reduces low-density lipoprotein and cholesterol levels and increases the level of high-density lipoprotein. Wilson and coworkers ${ }^{37}$ studied the relationship between estrogen therapy and cardiovascular disease in postmenopausal women participating in the Framingham Heart Study. They concluded that the risk of cardiovascular morbidity and stroke is higher among estrogen users. They also reported an increased incidence of myocardial infarction among estrogen users who smoke cigarettes. On the other hand, Stampfer and associates ${ }^{38}$ reported that estrogen therapy in postmenopausal women reduced the risk of severe coronary heart disease.

At the present time, it is difficult to define the effect of estrogen on the cardiovascular system. Further studies are needed to obtain more definitive information regarding potential protective or deleterious cardiovascular effects of estrogen.

\section{Fluoride use}

The predominant effect of fluoride is to increase bone production by stimulating osteoblasts. Fluoride therapy increases trabecular bone mass, but it does not increase and possibly decreases cortical bone mass. Giving fluoride to calcium-deprived animals resulted in increased bone mass but decreased bone strength. ${ }^{39}$ Fluoride replaces calcium hydroxyapatite crystals, which leads to increased crystallinity and decreased elasticity. Riggs and coauthors ${ }^{40}$ reported significant decreases in vertebral fractures in postmenopausal osteoporosis when fluoride was added to calcium and estrogen therapy.

Currently, the FDA has not approved the use of sodium fluoride for use in postmenopausal osteoporosis, and this treatment is still experimental. The optimum dosage and duration of therapy have not yet been determined, but daily doses of 40 to $80 \mathrm{mg}$ /day have been used. ${ }^{24}$ Daily fluoride dosage should be adjusted on the basis of serum fluoride values: Therapeutic range, 5 to $15 \gamma$ at 24 hours after last dose. One to $1.5 \mathrm{~g}$ of elemental calcium should be used with fluoride to decrease the incidence of osteomalacia.

The primary action of fluoride is on axial skeleton; therefore, it is most useful for patients with vertebral crush fractures. One-third of patients receiving fluoride therapy can be expected to develop side effects (Table 9). Patients with impaired renal function are not good candidates for fluoride therapy because of the increased risk of toxicity.

O'Duffy and colleagues ${ }^{41}$ studied patients with acute lower extremity pain who were receiving fluoride therapy for osteoporosis. They found that all patients with pain had abnormal bone scans, and that half of those had stress microfractures. They 
attributed the pain to intense regional bone remodeling, which may become complicated by stress fractures, rather than to fasciitis and synovitis.

\section{Calcitonin therapy}

Increasing serum calcium promotes calcitonin secretion, while a low calcium level inhibits its secretion. Calcitonin seems to compete with the effect of PTH on bone; therefore, it inhibits resorption of the bone by PTH. Calcitonin acutely inhibits osteoclast function and decreases their numbers over long-term administration (Table 10). On this basis, it is used in treatment of postmenopausal osteoporosis, Paget's disease of the bone, and hypercalcemia. It has been shown that estrogen therapy raises the level of calcitonin in postmenopausal women.

There has been an increasing interest in the use of this hormone in the treatment of osteoporosis. Calcitonin is reported ${ }^{42}$ to be effective in the relief of pain and in improving functional impairment of patients with osteoporosis. It is believed that the analgesic effect of calcitonin is related to an endogenous opiate, $\beta$-endorphin. Administration of 100 MRC units daily ${ }^{43}$ or every other day ${ }^{44}$ of salmon calcitonin in postmenopausal osteoporatic women demonstrated an increase in total body calcium and bone mineral content.

The usual dosage of synthetic salmon calcitonin is $100 \mathrm{MRC}$ units injected subcutaneously daily or every other day. Because it causes hypercalciuria and decreases calcium mobilization from bone, it may cause hypocalcemia and secondary stimulation of PTH. Therfore, daily calcium supplements of 1 to $1.2 \mathrm{~g}$ should be used along with the calcitonin.

The most common side effects of calcitonin are a warm flush and nausea, which can be minimized by injection at bedtime or use of antihistamine, such as 25 to $50 \mathrm{mg}$ diphenhydramine 30 minutes before injection. Anticalcitonin antibody developed in about $50 \%$ of patients with Paget's disease of bone treated with salmon calcitonin, but clinical response was not diminished. ${ }^{45}$ The investigator concluded that antibody formation does not affect the therapeutic efficacy of salmon calcitonin. Calcitonin has been used also for the osteoporosis of Sudeck's atrophy and for pain of neurogenic claudication. There is no information on long-term benefits of calcitonin treatment and its effect on rate of fracture.

\section{Management of acute vertebral crush fracture syndrome}

Compression fractures of dorsal and lumbar vertebrae are common in postmenopausal women. They may occur in the absence of trauma. The pain usu-
TABLE 9. ADVERSE REACTIONS TO FLUORIDE THERAPY.

\section{Arthralgia}

Painful plantar fasciitis

Anorexia, nausea, bloating

Constipation, diarrhea, peptic ulcer

TABLE 10. ACTIONS OF CALCITONIN.

Inhibits the release of calcium from bone by:

Decreasing activity and reducing the number of osteoclasts.

Facilitating transport and sequestration of calcium from extracellular fluid to bone cells fluid.

Increases urinary calcium.

Stimulates formation of 24, 25-dihydroxycholecalciferol in the kidney.

ally is acute and may persist for several weeks. The following regimen is recommended for these patients: First, bed rest (usually for 7 to 14 days) is necessary. Supine position on a firm mattress is preferred. Extra care of the skin is recommended to prevent pressure sores and a pillow under the knees decreases stress on the low-back area.

Second, analgesics and muscle relaxants should be used to relieve the pain and spasm of paraspinal muscle.

Third, measures should be taken to avoid constipation, urinary retention, dehydration, and venous thrombosis. Paralytic ileus may occur with higher lumbar fractures.

Fourth, when the acute phase resolves, usually 7 to 14 days after the initial event, mobilization begins with sitting or standing positions for 15 to 20 minutes several times daily. The frequency and duration are increased gradually. Patients with mid-dorsal or lower dorsal vertebral fractures may benefit from a rigid or semirigid thoracolumbar hyperextension orthosis.

Fifth, as soon as the acute pain resolves, the patient should begin active spinal extension exercises. Deep breathing exercises and pectoral stretching are recommended in patients with midthoracic compression. The use of a cane is recommended, particularly for elderly patients who might have a problem with coordination. Lifting and bending should be avoided. Usually after six to eight weeks, the patient can resume activities at the prefracture level.

\section{Summary}

Osteoporosis is the result of an imbalance between the rate of bone resorption and bone formation. The loss of bone mass which progress with age is markedly accelerated in postmenopausal females and in- 
creases the risk of fracture. Prevention of this disease is the only cost-effective approach. Education of adolescents on better nutrition, adequate physical activity, and avoidance of smoking to optimize bone mass during growth, and prophylactic treatment of high-risk postmenopausal women will decrease the incidence of osteoporosis and its complications.

1. Gallagher JC, Riggs BL, Eisman J, et al: Intestinal calcium absorption and serum vitamin D metabolites in normal subjects and osteoporotic patients: Effects of age and dietary calcium. $J$ Clin Invest 1979;64:729-736.

2. Slovik DM, Adams JS, Neer RM, et al: Deficient production of 1,25 dihydroxy-vitamin $\mathrm{D}$ in elderly osteoporotic patients. $N$ Engl $J$ Med 1981;305:372-374.

3. Hillyard CJ, Stevenson JC, MacIntyre I: Relative deficiency of plasma calcitonin in normal women. Lancet 1978;1:961-962.

4. Deftos LJ, Weisman MH, Williams GW, et al: Influence of age and sex on plasma calcitonin in human beings. $N$ Engl J Med 1980; 302:13511353.

5. Hurley DL, Tiegs RD, Wahner HW, et al: Axial and appendicular bone mineral density in patients with long-term deficiency or excess of calcitonin. N Engl J Med 1987;317:537-541.

6. Heaney RP, Recker RR, Saville PD: Calcium balance and calcium requirements in middle-aged women. Am J Clin Nutr 1977;30:1603. 1611.

7. Heaney RP, Recker RR, Saville PD: Menopausal changes in calcium balance performance. $J$ Lab Clin Med 1978;92:953-963.

8. Schuette SA, Zemel MB, Linkswiler HM: Studies on the mechanism of protein-induced hypercalciuria in older men and women. $J$ Nutr 1980;110:305-315.

9. Jensen J, Christiansen C, Rodbro P: Cigarette smoking, serum estrogens, and bone loss during hormone-replacement therapy early after menopause. N Engl J Med 1985;313:973-975.

10. Hart P, Farrell GC, Cooksley WGE, et al: Enhanced drug metabolism in cigarette smokers. Br Med $J$ 1976;2:147-149.

11. Coindre JM, David JP, Riviere L, et al: Bone loss in hypothyroidism with hormone replacement: A histomorphometric study. Arch Intern Med 1986;146:48-53.

12. Dequeker J: The relationship between osteoporosis and osteoarthritis. Clin Rheum Dis 1985;11:271-296.

13. Drimkwater BL, Nilson K, Chestnut III, CH, et al: Bone mineral content of amenorrheic and eumenorrheic athletes. N Engl J Med 1984; 311:277-281.

14. Kroølner B, Toft B, Vertebral bone loss: An unheeded side effect of therapeutic bed rest Clin Sci 1983;64:537-540.

15. Maltovic V, Costial R, Simonovic I, et al: Bone status and fracture rate in two regions of Yugoslavia. Am J Clin Nutr 1979;32:540-549.

16. Riggs BL, Wahner HW, Melton LJ III, et al: Dietary calcium intake and rate of bone loss in women. J Clin Invest 1987;80:979-982.

17. Baker MR, Peacok M, Nordin BEC: The decline in vitamin D status with age. Age Ageing 1980;9:249-252.

18. Tsai K-S, Heath H III, Kumar R, et al: Impaired vitamin D metabolism with aging in women: Possible role in pathogenesis of senile osteoporosis. J Clin Invest 1984;73:1668-1672.

19. Eriksen EF, Berg NJ, Graham ML, et al: Evidence of estrogen receptors in human bone cells, abstract 238. J Bone Mineral Res 1987;2:supp. 1, Jun 1987.

20. Lindsay R, Hart DM, Purdie D, et al: Comparative effects of estrogen and progestogen on bone loss in postmenopausal women. Clin Sci 1978; 54:193-195.

21. Lindsay R, Hart DM, Aitken JM, et al: Long-term prevention of postmenopausal osteoporosis by estrogen: Evidence for an increased bone mass after delayed onset of estrogen treatment. Lancet 1976;1:10381040.

22. Lindsay R, Hart DM, MacLean A, et al: Bone response to termination of estrogen treatment. Lancet 1978;1:1325-1327.
23. Lindsay, R, Hart DM, Forrest C, et al: Prevention of spinal osteoporosis in oophorectomized women. Lancet 1980;2:1151-1154.

24. Riggs BL, Mefton III, J: Osteoporosis: Etiology, diagnoses, and management. New York, Raven Press, 1988, pp333-358.

25. Ettinger B, Genant HK, Cann CE: Postmenopausal bone loss is prevented by treatment with low-dose estrogen with calcium. Ann Intern Med 1987;106:40-45.

26. Antunes CMF, Stolley PD, Rosenshein NB, et al: Endometrial cancer and estrogen use: Report of a large case control study. $N$ Engl $J$ Med 1979;300:9-13.

27. Piziak V, Shull BL: Menopausal hormone therapy. Hosp Prac 1985;20:82bb-82rr.

28. Kaufman DW, Shapiro S, Slone D, et al: Decreased risk of endometrial cancer among oral contraceptive users. N Engl J Med 1980;303:10451047.

29. Hulka BS, Kaufman DG, Fowler Jr., WC, et al: Predominance of early endometrial cancers after long-term estrogen use. JAMA $1980 ; 244: 2419-2422$.

30. Jick H, Watkins RN, Hunter JR, et al: Replacement estrogens and endometrial cancer. $N$ Engl $J$ Med 1979;300:218-222.

31. Mack TM, Pike MC, Henderson BE, et al: Estrogens and endometrial cancer in a retirement community. $N$ Engl J Med 1976;294:12621267.

32. Shapiro S, Kelly JP, Rosenberg L, et al: Risk of localized and widespread endometrial cancer in relation to recent and discontinued use of conjugated estrogens. $N$ Engl J Med 1985; 313:969-972.

33. Surgically confirmed gallbladder disease, venus thromboembolism, and breast tumors in relation to postmenopausal estrogen therapy: A report from the Boston Collaborative Drug Surveillance. N Engl J Med 1974; 290:15-19.

34. Oral contraceptive use and the risk of breast cancer: The Cancer and Steroid Hormone Study of the Centers for Disease Control and the National Institute of Child Health and Human Development. $N$ Engl $J$ Med 1986;315:405-411.

35. Alkjaersig N, Fletcher A, Burstein R: Association between oral contraceptive use and thromboembolism: A new approach to its investigation based on plasma fibrinogen chromatography. Am J Obstet Gynecol 1975;122:199-211.

36. Notelvitz M, Kitchens C, Ware M, et al: Combination estrogen and progestogen replacement therapy does not adversely affect coagulation. Obstet Gynecol 1983;62:596-600.

37. Wilson PWF, Garrison RJ, Castelli, WP: Postmenopausal estrogen use, cigarette smoking, and cardiovascular morbidity in women over 50: The Framingham study. N Engl $J$ Med 1985;313:1038-1043.

38. Stampfer MJ, Willett WC, Colditz GA, et al: A prospective study of postmenopausal estrogen therapy and coronary heart disease. $N$ Engl $J$ Med 1985;313:1044-1049.

39. Riggs RS, Rucker RC, Chan MM: The effect of fluoride supplementation on the strength of osteopenic bone. Clin Orthop 1976;223:352-357. 40. Riggs BL, Seeman E, Hodgson SF, et al: Effect of fluoride-calcium regimen on vertebral fracture occurrence in postmenopausal osteoporosis. $N$ Engl $J$ Med 1982;306:446-450.

41. O'Duffy JD, Wahner HW, O'Fallon WM, et al: Mechanism of acute lower extremity pain syndrome in fluoride-treated osteoporotic patients. Am J Med 1986;80:561-566.

42. Maresea V: Human calcitonin in the management of osteoporosis. $J$ Intern Med Res 1985;13:311-316.

43. Gruber HE, Ivey JL, Baylink DJ, et al; Long-term calcitonin therapy in postmenopausal osteoporosis. Metabolism 1984;33:295-303.

44. Mazzuoli GF, Passer, M, Gennari C, et al: Effects of salmon calcitonin therapy in postmenopausal osteporosis: A controlled double-blind clinical study. Calcif Tissue Int 1986;38:3-8.

45. Hosking DJ: Functional significance of antibody formation after longterm salmon calcitonin therapy. Clin Endocrinol 1979;10:243-252.

From the Department of Medicine, Section of Rheumatology, Chicago College of Osteopathic Medicine, Chicago.

Reprint requests to Dr. Hedayati, 5200 S. Ellis Ave, Chicago, 60615. 